\title{
Does aging change docosahexaenoic acid homeostasis? Implications for the challenge to cognitive health in the elderly
}

\author{
Christian-Alexandre \\ CASTELLANO ${ }^{1,4}$ \\ Raphaël CHOUINARD-WATKINS ${ }^{1,4}$ \\ J. Tom BRENNA ${ }^{2}$ \\ Barbara J. MEYER ${ }^{3}$ \\ Stephen C. CUNNANE ${ }^{1,4}$ \\ ${ }^{1}$ Research Center on Aging, Université \\ de Sherbrooke, 1036 Belvedere Sud, \\ Sherbrooke, QC, Canada J1H 4C4 \\ $<$ Stephen.Cunnane@USherbrooke.ca> \\ ${ }^{2}$ Division of Nutritional Sciences, \\ Cornell University, Ithaca, NY, USA \\ ${ }^{3}$ Metabolic Research Centre, School of \\ Health Sciences, University of \\ Wollongong NSW 2522, Australia \\ ${ }^{4}$ Departments of Medicine and \\ Physiology and Biophysics, Université de \\ Sherbrooke, 3001, 12e Avenue Nord, \\ Sherbrooke, QC, Canada J1H 5N4
}

\section{DHA and aging-related cognitive decline}

Cognitive health in the elderly is an increasingly important preoccupation for public health institutions globally. This preoccupation is all the more acute given the lack of effective medical treatment for the various types of cognitive decline associated with aging including "Alzheimer's disease". Because the various forms of cognitive decline in the elderly are increasingly recognised as a heterogeneous conditions (Whitehouse et al., 2011), we prefer the general term aging-related cognitive decline (ARCD). Conditions linked to the metabolic syndrome, particularly insulin resistance and type 2 diabetes, are major risk factors for

\begin{abstract}
Epidemiological studies fairly convincingly suggest that higher intake of fish and omega-3 fatty acids present in fish is associated with reduced risk for age-related cognitive decline (ARCD). Normally, docosahexaenoic acid (DHA) in plasma is positively associated with DHA intake. However, despite being associated with lower fish and DHA intake, unexpectedly, $A R C D$ is not consistently associated with lower plasma DHA. Furthermore, DHA is often slightly but significantly higher in plasma and erythrocytes in the elderly without ARCD compared to young adults. Higher plasma DHA in the elderly may be a sign that their fish or DHA intake is higher but we show here that various aspects of DHA homeostasis also change with age. Our supplementation and tracer studies show that DHA metabolism, e.g. transit through the plasma and apparent retroconversion but not beta-oxidation, is different in healthy elderly compared to healthy young adults. Apolipoprotein E4 increases the risk of $A R C D$, possibly in part because it changes DHA homeostasis. Therefore, independent of differences in fish intake, changing DHA homeostasis may contribute to making the elderly more susceptible to cognitive decline despite them having similar or sometimes higher plasma DHA than in younger adults.
\end{abstract}

Key words: aging, cognitive decline, dietary, docosahexaenoic acid, omega-3 fatty acid

cognitive decline in the elderly (Hao et al., 2011). Since the metabolic syndrome is preventable at least in adolescents and younger adults, in theory, prevention should be able to play a key role in the reducing the risk of ARCD. Nutrition and physical activity are key elements of prevention in reducing the susceptibility to the metabolic syndrome and type 2 diabetes. Omega- 3 fatty acids such as docosahexaenoic acid (DHA) are amongst the nutrients of particular interest relative to optimal glucose metabolism, especially for cognition (Cunnane et al., 2009; Pifferi et al., 2010; Cunnane et al., 2011).

Our aim here is to draw attention to an apparent dichotomy: On the one hand, there is the relatively solid evidence that low fish and low DHA intake are common in ARCD; on the other hand, there is the lack of consistent evidence that brain or plasma omega-3 fatty acids, particularly $D H A$, are any different in ARCD than in age-matched controls (Cunnane et al., 2009). Furthermore, some studies show a benefit on cognitive function of higher intake of fish or DHA in the elderly (Beydoun et al., 2007; Dullemeijer et al., 2007; Yurko-Mauro et al., 2010), but others did not observe any difference in cognitive performance (Van De Rest et al., 2008; Dangour et al., 2010). These observations seem contradictory and counter-intuitive: Why is it that if fish intake is low in ARCD, and if fish intake is an important predictor of plasma DHA,

To cite this article: Castellano CA, Chouinard-Watkins R, Brenna JT, Meyer BJ, Cunnane SC. Does aging change docosahexaenoic acid homeostasis? Implications for the challenge to cognitive health in the elderly. OCL 2011; 18(4): 175-80. doi : 10.1684/ocl.2011.0390 
that plasma DHA is not more consistently reported to be lower in ARCD? Equally if not more importantly, can omega-3 supplements correct an apparent DHA deficit in the elderly and thereby reduce the risk of ARCD and, if so, under what conditions, because presently the outcomes of such studies have not been too promising (Freund-Levi et al., 2006; Fotuhi et al., 2009; Quinn et al., 2010).

We have previously raised some methodological issues that may contribute to this apparent dichotomy between the epidemiological versus the biological evidence for a protective role of fish and/or DHA in ARCD (Cunnane et al., 2009) and will discuss here some potential biological reasons for this inconsistency. Our focus here is not so much on ARCD per se but on the healthy elderly because clear indications are emerging that omega-3 fatty acid, at least DHA, metabolism changes in the elderly with no symptoms of ARCD.

\section{Plasma DHA response to a DHA-rich supplement}

We and others have observed that the healthy elderly tend to have 10-15\% higher DHA and eicosapentaenoic acid (EPA) in plasma (Dewailly et al., 2003; Crowe et al., 2008; Fortier et al., 2010; Yanagisawa et al., 2010) and red blood cells (De Groot et al., 2009), but there are exceptions (Babin et al., 1999; Sfar et al., 2010). Somewhat higher plasma DHA may be due in part to higher DHA intakes in the elderly (Meyer et al., 2003; Buyken et al., 2010). Given that we have not tracked DHA intakes in our elderly study participants (Fortier et al., 2010), we did not know whether the higher plasma EPA and DHA were due to higher fish and seafood intake in the elderly or to an aging-related change in DHA metabolism. We decided to probe the possibility of an aging-related change in DHA metabolism with a simple three week supplementation study in which participants received about $680 \mathrm{mg}$ $\mathrm{DHA}$ /day, which is about 4-5 times usual intake in many developed countries (Kris-Etherton et al., 2009; Lucas et al., 2010; Meyer, 2011). Participants were screened to eliminate those already consuming high amounts of fish or fish oil supplements. Interestingly, despite almost identical fasting DHA at baseline, after 3 weeks supplementation, the elderly participants had a $42 \%$ higher plasma response to the same supplement as the young participants (figure 1) (Vandal et al., 2008). This was a preliminary indication to us that perhaps DHA metabolism was changing with healthy aging, e.g. independently of ARCD.

\section{Carbon-13-labelled DHA transit through plasma}

The fish oil supplementation study (Vandal et al., 2008) led us to examine metabolism of carbon-13 $\left({ }^{13} \mathrm{C}\right)-\mathrm{DHA}$ in the healthy elderly. Again, we were surprised to see a very significant difference in the transit of a single oral $50 \mathrm{mg}$ dose of ${ }^{13} \mathrm{C}-\mathrm{DHA}$ through the plasma with repeated blood sampling over a 4 week study period. The elderly had slower DHA transit in the form of higher ${ }^{13} \mathrm{C}$-DHA enrichment in all plasma lipid pools examined. This slower clearance was observed in the pools rapidly labeled in the first hours after the oral dose, ex. free fatty acids and triglycerides, as well as in those pools increasingly labeled days to weeks later, ex. phospholipids and cholesteryl esters (figure 2) (Plourde et al., 2011). These results support the idea that higher plasma
DHA (fasting or response to a fish oil supplement) in the elderly is not necessarily an indication of "better" DHA metabolism, better DHA status or even higher intake of DHA; it may well be that aging changes DHA homeostasis in a way that slows down its transfer to tissues including the brain thus increasing its levels in plasma, at least transiently. This apparent delay in DHA transport into tissues may underlie and contribute to the increased susceptibility to cognitive decline in the elderly.

\section{Other components of homeostasis}

${ }^{13} \mathrm{C}-\mathrm{DHA}$ provides an opportunity to measure other components of DHA homeostasis besides plasma levels, including beta-oxidation, retroconversion, and conversion to bioactive metabolites. One day after an oral dose of ${ }^{13} \mathrm{C}$-DHA, about $7 \%$ of it is completely beta-oxidized to $\mathrm{CO}_{2}$ (Plourde et al., 2011), which is about $30 \%$ of the betaoxidation of the same dose of ${ }^{13} \mathrm{C}$-linoleic acid and about $25 \%$ of that of ${ }^{13} \mathrm{C}$-alphalinolenic acid given under the same conditions. Seven days after giving the oral dose of ${ }^{13} \mathrm{C}-\mathrm{DHA}$, about $35 \%$ is beta-

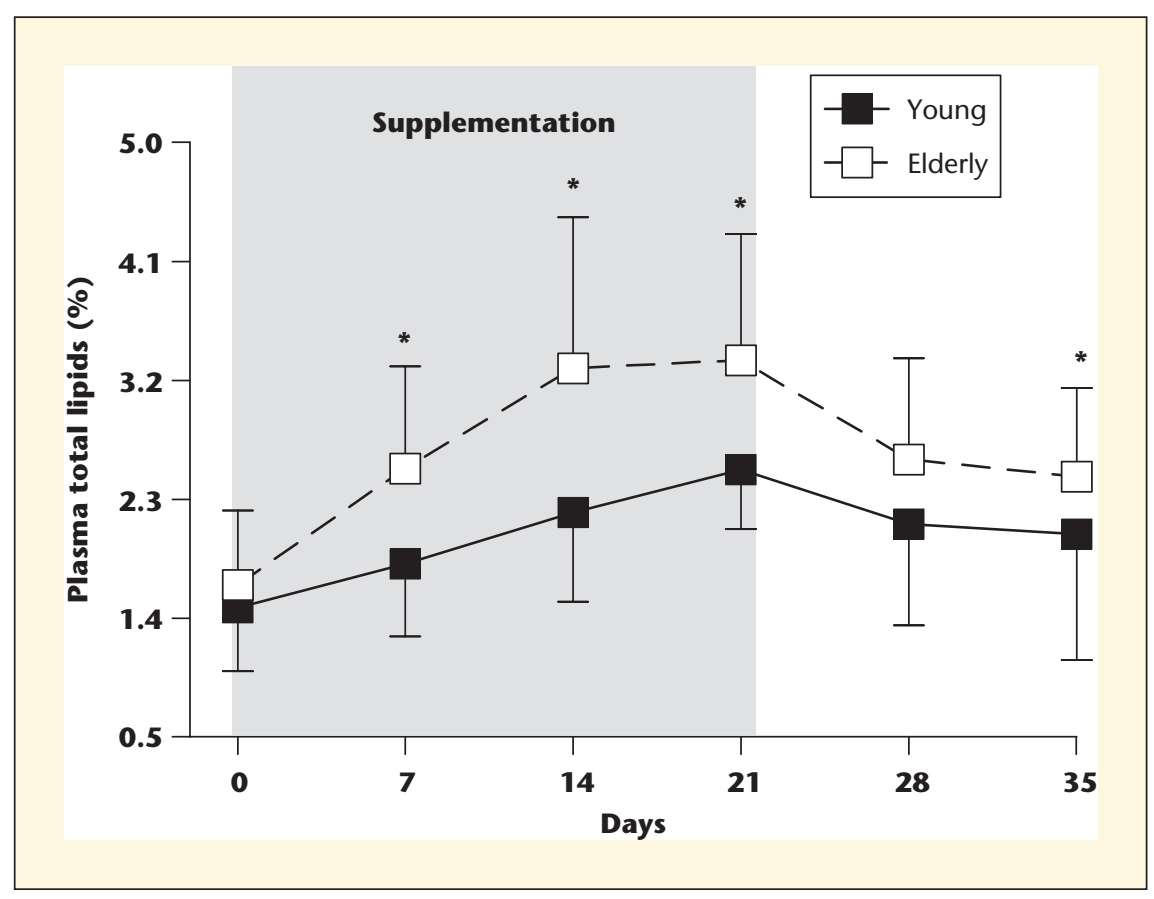

Figure 1. DHA in plasma total lipids in healthy young and elderly humans ( $24 \pm 2$ and $74 \pm 4$ years old, respectively) during a 3 week dietary supplementation with fish providing $680 \mathrm{mg} / \mathrm{d}$ DHA, as adapted from Vandal et al (2008). At the end of the supplementation period, plasma $D H A$ was $42 \%$ higher in the elderly group than in the young group $\left.{ }^{*} p<0.05\right)$. 


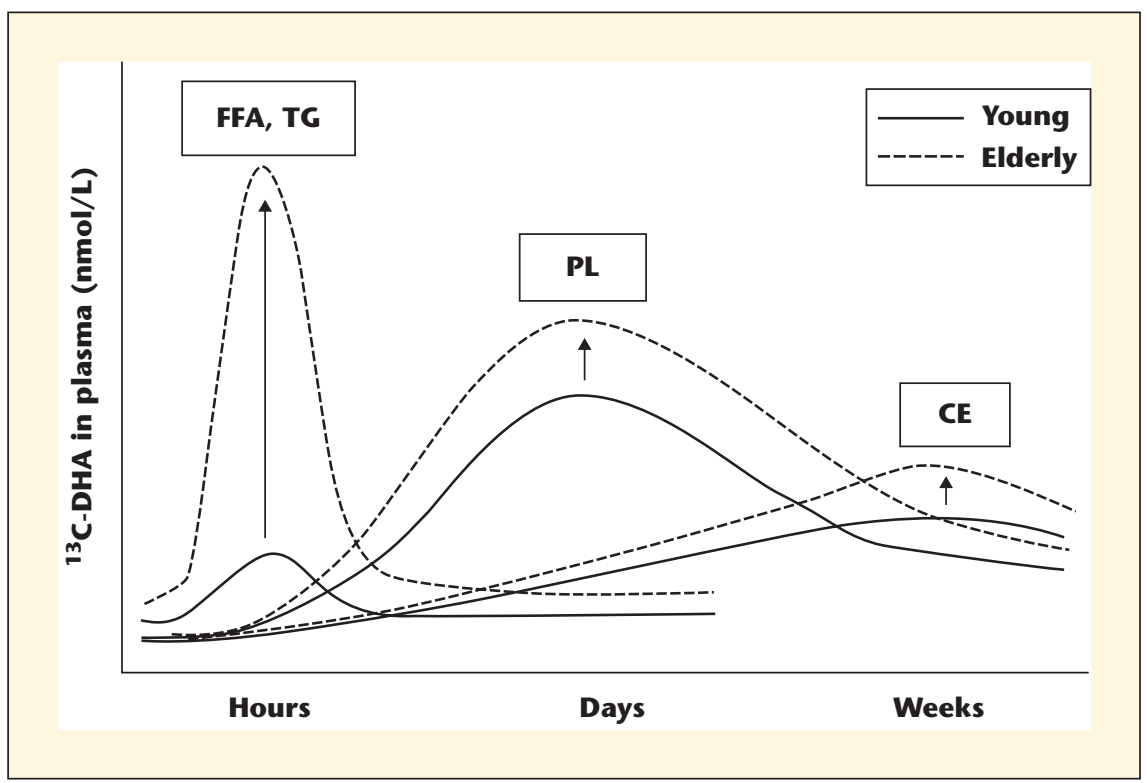

Figure 2. Healthy aging delays plasma clearance of ${ }^{13} \mathrm{C}-\mathrm{DHA}$ as adapted from Plourde et al. (2011). In the elderly, plasma tracer concentration in free fatty acids (FFA) and triglycerides (TG) was 4-5 fold higher $4 \mathrm{~h}$ after giving the $50 \mathrm{mg}$ oral dose whereas in phospholipids (PL) and cholesteryl esters (CE), ${ }^{13} \mathrm{C}$-DHA was 2-fold higher 1-4 weeks later (Young: $26.8 \pm 2.6$ years ; Elderly; $76.5 \pm 2.7$ years).

oxidized (figure 3 ), whereas ${ }^{13} \mathrm{C}$-linoleic acid and ${ }^{13} \mathrm{C}$-alpha-linolenic acid are beta-oxidized at $40 \%$ and $70 \%$, respectively (McCloy et al., 2004). Up to seven days post-dose, beta-oxidation of ${ }^{13} \mathrm{C}$ DHA does not seem to be affected by healthy aging. However, the apparent

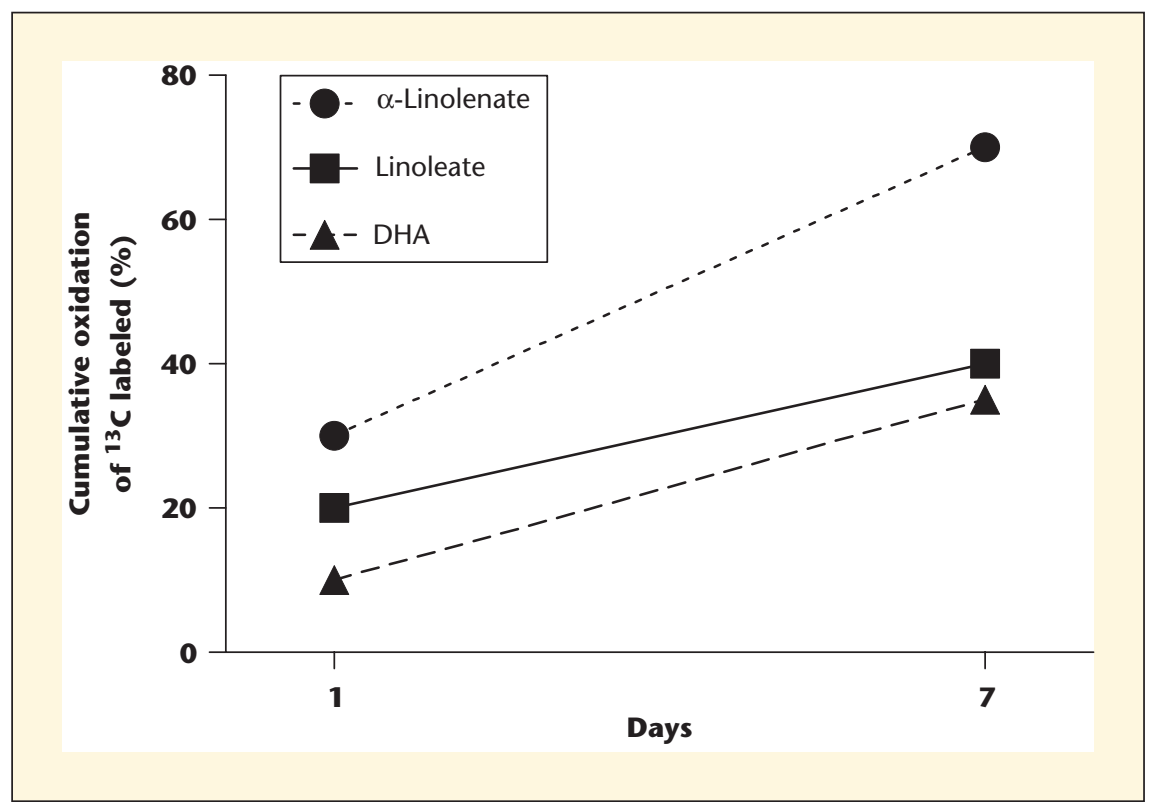

Figure 3. Slower beta-oxidation of ${ }^{13} \mathrm{C}$-docosahexaenoic acid $(\mathrm{DHA})$ compared to ${ }^{13} \mathrm{C}$-linoleate and ${ }^{13} \mathrm{C}$ - $\alpha$-linolenate over 1 and $7 \mathrm{~d}$. Adapted from McCloy et al. (2004) and Plourde et al. (2011).

\section{Genotype (apo E)}

Whalley et al. (2008) were the first to suggest that apolipoprotein (apo) E genotype might affect DHA metabolism; they reported that cognitive decline was associated with lower erythrocyte omega-3 fatty acid content only in the presence of the apo E4 allele. We have also observed that apo E genotype influences plasma EPA and DHA after a fish oil supplement, with apo E4 significantly reducing the rise in plasma DHA after 6 wk of fish oil supplementation that provided $1.9 \mathrm{mg} / \mathrm{d}$ of EPA and $1.1 \mathrm{mg} / \mathrm{d}$ of DHA (Plourde et al., 2009).

We have not yet assessed the relationship between DHA metabolism and apo E genotype in the elderly but given that apo E4 predisposes to various forms of ARCD (Song et al., 2009; Leoni, 2011), this interaction bears further scrutiny. As suggested by Whalley et al. (2008), one potential explanation of a relation between omega- 3 fatty acids and apo $E$ genotype would be increased oxidative stress that catabolises DHA. Studies in vitro (Miyata and Smith, 1996; Jolivalt et al., 2000) and in mice (Ramassamy et al., 2000) and humans (Kharrazi et al., 2008) suggest that apo E4 carriers have greater susceptibility to oxidative damage and lower antioxidant defenses.

\section{Relation between dietary and plasma DHA}

If lower habitual fish and/or DHA intake increase the risk of ARCD (reviewed by Cunnane et al., 2009) but this is not reflected by lower plasma DHA in ARCD, then the relation between dietary and plasma DHA in the elderly and in ARCD needs further attention. Our preliminary assessment of several published papers on this topic shows the anticipated and relatively consistent positive, dosedependent relation between DHA intake and plasma DHA (figure 4) (Blonk et al., 1990). We believe that a crucial point needing verification is whether ARCD is associated with a leftward shift in the diet-plasma relationship for DHA (figure 5). This possibility is suggested by at least three observations: (i) the lack of consistently lower plasma DHA in the elderly with ARCD (Cunnane et al., 2009), (ii) the higher plasma DHA and ${ }^{13} \mathrm{C}$-DHA response in the healthy elderly (Vandal et al., 2008; Plourde et al., 2011), and (iii) the similar plasma DHA levels but 


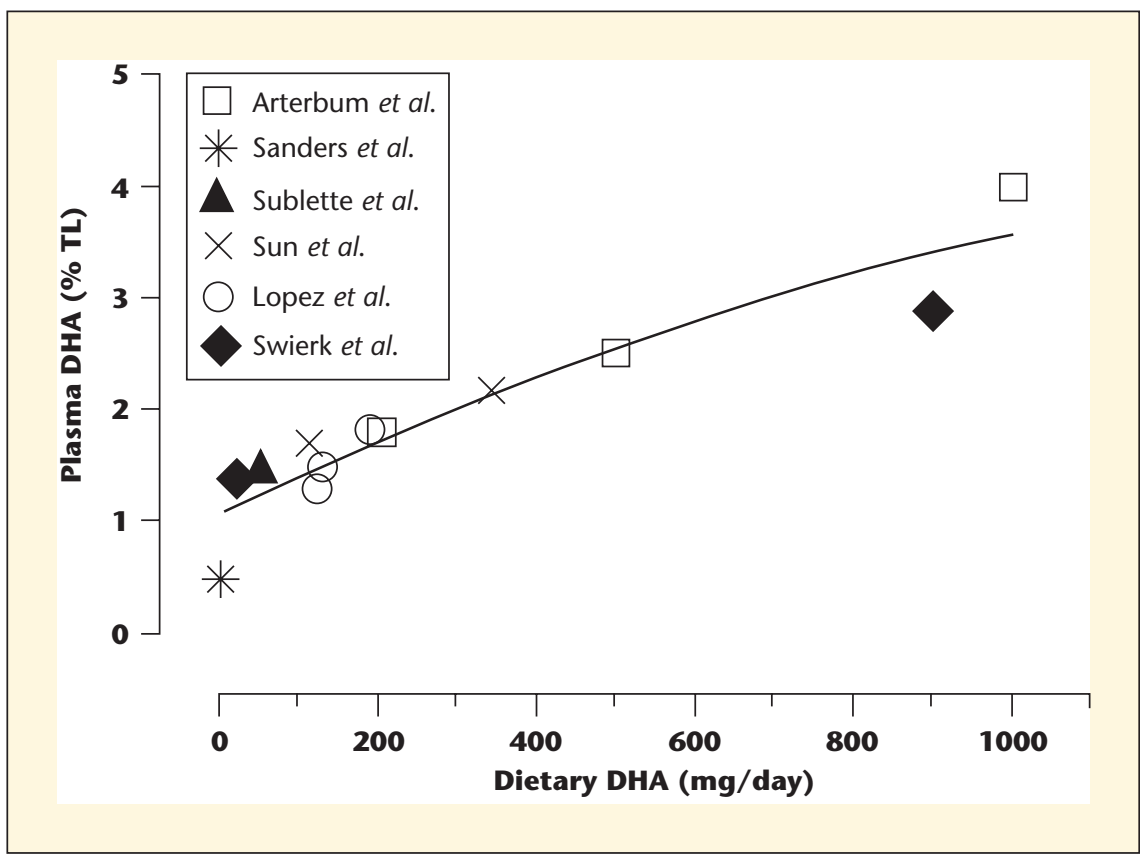

Figure 4. Near linear relation between dietary and plasma DHA at DHA intakes below $1000 \mathrm{mg} / \mathrm{d}$ (Sanders et al., 1989; Arterburn et al., 2006; Sublette et al., 2007; Sun et al., 2007; Lopez et al., 2010; Swierk et al., 2011).

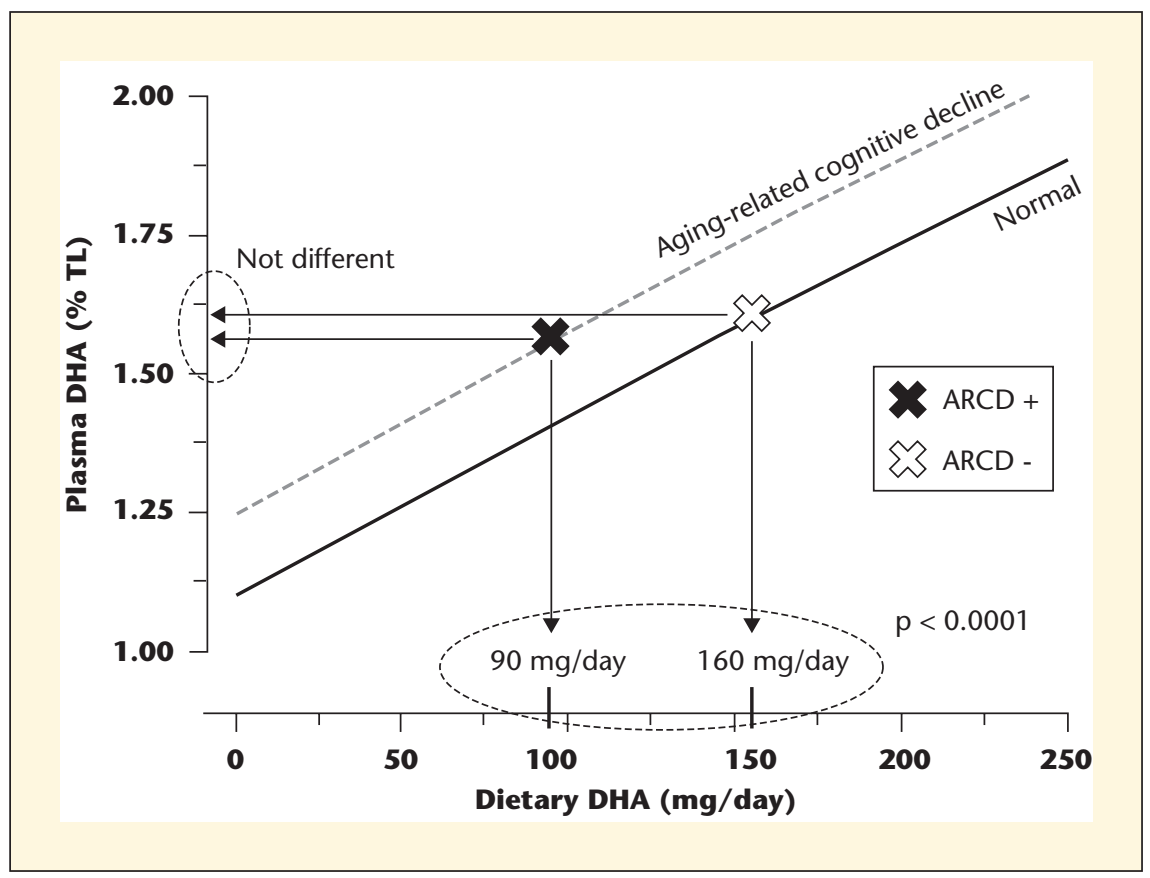

Figure 5. Dietary verses plasma DHA in aging-related cognitive decline $(A R C D)$, based on Lopez et al (2010). In Lopez et al. (2010), daily intake of $90 \mathrm{mg}$ DHA in the elderly was associated with cognitive decline (ARCD+), whereas $160 \mathrm{mg}$ of DHA was associated with an absence of cognitive decline (ARCD-), yet \% DHA in plasma total lipids was not significantly different in the two groups. Though by no means conclusive, these data support our hypothesis that there may be a shift in DHA homeostasis in the elderly with ARCD. lower DHA intake in those with ARCD (Lopez et al., 2010). We see this as a topic for fruitful investigation and, at the moment, draw no firm conclusion as to whether this key component of DHA homeostasis, i.e. plasma response to habitual dietary DHA intake, is altered by healthy aging or cognitive decline in the elderly.

\section{Conclusion}

Although both prospective and crosssectional epidemiological studies clearly associate ARCD with lower fish and DHA intake, ARCD is not consistently associated with lower plasma DHA (Cunnane et al., 2009). Indeed, DHA is often slightly but significantly higher in plasma and erythrocytes in the elderly compared to young adults even though the former are at increased risk of ARCD. Higher plasma DHA in the elderly is not necessarily a sign that their fish intake is higher; we review here several of our studies showing that DHA homeostasis appears to change with age. Our tracer and supplementation studies show that DHA metabolism, i.e. transit through the plasma and apparent retroconversion, changes with aging in the absence of ARCD. Apo E4 increases the risk of $A R C D$, and our studies show that this may be in part because it changes DHA homeostasis. Independent of differences in fish intake, changing DHA homeostasis may contribute to making the elderly more susceptible to cognitive decline despite them having similar or even somewhat higher plasma DHA than in younger adults. Identifying how and why DHA homeostasis changes with age may contribute to resolving the problems with clinical trials that have unsuccessfully used fish oil or DHA supplements to treat ARCD.

Acknowledgements. Funding was provided by the Natural Science and Engineering Research Council of Canada, Canadian Institutes of Health Research, Canadian Foundation for Innovation and Canada Research Chairs Secretariat. Excellent assistance was provided by Jennifer Tremblay-Mercier, Mélanie Fortier and Conrad Filteau. The collaboration of Mélanie Plourde in the projects leading to this review is greatly appreciated.

\section{REFERENCES}

Arterburn LM, Hall EB, Oken H. Distribution, interconversion, and dose response of $n-3$ 
fatty acids in humans. Am / Clin Nutr 2006; 83: 1467S-76S.

Babin F, Abderrazik M, Favier F, et al. Differences between polyunsaturated fatty acid status of non-institutionalised elderly women and younger controls: A bioconversion defect can be suspected. Eur / Clin Nutr 1999; 53: 591-6.

Beydoun MA, Kaufman JS, Satia JA, Rosamond W, Folsom AR. Plasma n-3 fatty acids and the risk of cognitive decline in older adults: The atherosclerosis risk in communities study. Am J Clin Nutr 2007; 85: 1103-11.

Blonk MC, Bilo HJG, Nauta JJP, Popp-Snijders C, Mulder C, Donker AJM.Dose-response effects of fish-oil supplementation in healthy volunteers. Am J Clin Nutr 1990; 52: $120-7$.

Buyken A, Flood V, Rochtchina E, Nestel P, Brand-Miller I, Mitchell P. Modifications in dietary fat quality are associated with chnages in serum lipids of older adults independently of lipid medication. I Nutr 2010; 140: 88-94.

Crowe FL, Skeaff CM, Green TJ, Gray AR. Serum n-3 long-chain PUFA differ by sex and age in a population-based survey of $\mathrm{New}$ Zealand adolescents and adults. Br J Nutr 2008; 99: 168-74.

Cunnane S, Nugent S, Roy M, et al. Brain fuel metabolism, aging, and Alzheimer's disease. Nutrition 2011; 27: 3-20.

Cunnane SC, Plourde $M$, Pifferi F, Bégin $M$, Féart C, Barberger-Gateau P. Fish, docosahexaenoic acid and Alzheimer's disease. Prog Lipid Res 2009; 48: 239-56.

Dangour AD, Allen E, Elbourne D, et al. Effect of 2-y n-3 long-chain polyunsaturated fatty acid supplementation on cognitive function in older people: A randomized, double-blind, controlled trial. Am / Clin Nutr 2010; 91: 1725-32.

De Groot RHM, Van Boxtel MPJ, Schiepers OJG, Hornstra G, Jolles J. Age dependence of plasma phospholipid fatty acid levels: Potential role of linoleic acid in the age-associated increase in docosahexaenoic acid and eicosapentaenoic acid concentrations. Br / Nutr 2009; 102: 1058-64

Dewailly E, Blanchet C, Gingras S, Lemieux S, Holub BJ. Fish consumption and blood lipids in three ethnic groups of Québec (Canada). Lipids 2003; 38: 359-65.

Dullemeijer C, Durga J, Brouwer IA, et al. n-3 Fatty acid proportions in plasma and cognitive performance in older adults. Am J Clin Nutr 2007; 86: 1479-85.

Fortier M, Tremblay-Mercier J, Plourde M, et al. Higher plasma n-3 fatty acid status in the moderately healthy elderly in southern Québec: Higher fish intake or aging-related change in n-3 fatty acid metabolism? Prostaglandins Leukotrienes and Essential Fatty Acids 2010; 82: 277-80.

Fotuhi M, Mohassel P, Yaffe K. Fish consumption, long-chain omega-3 fatty acids and risk of cognitive decline or Alzheimer disease: A complex association. Nat Clin Pract Neurol 2009; 5: 140-52.

Freund-Levi $Y$, Eriksdotter-jönhagen $M$, Cederholm $\mathrm{T}$, et al. Omega-3 fatty acid treatment in 174 patients with mild to moderate Alzheimer disease: OmegAD study: a randomized double-blind trial. Arch Neurol 2006; 63: 1402-8.

Hao Z, Wu B, Wang D, Liu M. Association between metabolic syndrome and cognitive decline: A systematic review of prospective population-based studies. Acta Neuropsychiatrica 2011; 23: 69-74

Jolivalt C, Leininger-Muller B, Bertrand $P$, Herber R, Christen Y, Siest G. Differential oxidation of apolipoprotein $\mathrm{E}$ isoforms and interaction with phospholipids. Free Radical Biology and Medicine 2000; 28: 129-40.

Kharrazi H, Vaisi-Raygani A, Rahimi Z, Tavilani H, Aminian M, Pourmotabbed T. Association between enzymatic and nonenzymatic antioxidant defense mechanism with apolipoprotein $\mathrm{E}$ genotypes in Alzheimer disease. Clin Biochem 2008; 41: 932-6.

Kris-Etherton PM, Grieger JA, Etherton TD. Dietary reference intakes for DHA and EPA. Prostaglandins Leukotrienes and Essential Fatty Acids 2009; 81: 99-104.

Leoni V. The effect of apolipoprotein e (ApoE) genotype on biomarkers of amyloidogenesis, tau pathology and neurodegeneration in Alzheimer's disease. Clin Chem Lab Med 2011; 49: 375-83.

Lopez LB, Kritz-Silverstein D, Barrett-Connor E. High dietary and plasma levels of the omega- 3 fatty acid docosahexaenoic acid are associated with decreased dementia risk: The Rancho Bernardo study. I Nutr Health Aging 2010: 1-7.

Lucas M, Asselin G, Plourde M, Cunnane SC, Dewailly E, Dodin S.N-3 Fatty acid intake from marine food products among Quebecers: Comparison to worldwide recommendations. Public Health Nutrition 2010; 13: 6370.

McCloy U, Ryan MA, Pencharz PB, Ross RJ, Cunnane SC.A comparison of the metabolism of eighteen-carbon 13C-unsaturated fatty acids in healthy women. / Lipid Res 2004; 45: 474-85.

Meyer B. Are we consuming enough long chain omega-3 polyunstaurated fatty acids for optimal health? Prostaglandins Leukotrienes and Essential Fatty Acids. 2011.

Meyer B, Mann N, Lewis J, Milligan G, Sinclair A, Howe P. Dietary intakes and food sources of omeag- 6 and omega- 3 polyunsaturated fatty acids. Lipids 2003; 38: 391-8.

Miyata M, Smith J. Apolipoprotein E allelespecific antioxidant activity and effects on cytotoxicity by oxidative insults and betaamyloid peptides. Nat Genet 1996; 14: 55-61.

Pifferi $F$, Jouin M, Alessandri JM, et al. N-3 long-chain fatty acids and regulation of glucose transport in two models of rat brain endothelial cells. Neurochem Int 2010; 56: 703-10.

Plourde M, Chouinard-Watkins R, Vandal M, et al. Plasma incorporation, apparent retroconversion and $\beta$-oxidation of 13C-docosahexaenoic acid in the elderly. Nutrition and Metabolism 2011; 8.

Plourde M, Vohl MC, Vandal M, Couture P, Lemieux S, Cunnane SC. Plasma n-3 fatty acid response to an n-3 fatty acid supplement is modulated by apoE 4 but not by the common PPAR- L162V polymorphism in men. Br J Nutr 2009; 102: 1121-4.

Quinn JF, Raman R, Thomas RG, et al. Docosahexaenoic acid supplementation and cognitive decline in Alzheimer disease: A randomized trial. JAMA 2010; 304: 1903-11.

Ramassamy C, Averill D, Beffert U, et al. Oxidative insults are associated with apolipoprotein E genotype in Alzheimer's disease brain. Neurobiol Dis 2000; 7: 23-37.

Sanders TAB, Hinds A, Pereira CC. Influence of $\mathrm{n}-3$ fatty acids on blood lipids in normal subjects. J Intern Med, Supplement 1989; 225: 99-104.

Sfar S, Laporte F, Braham H, Jawed A, Amor S, Kerkeni A. Influence of dietary habits, age and gender on plasma fatty acids levels in a population of healthy Tunisian subjects. Experimental Gerontology 2010; 45: 719. 25.

Song F, Poljak A, Smythe GA, Sachdev P. Plasma biomarkers for mild cognitive impairment and Alzheimer's disease. Brain Res Rev 2009; 61: 69-80.

Sublette ME, Bosetti F, Demar JC, et al. Plasma free polyunsaturated fatty acid levels are associated with symptom severity in acute mania. Bipolar Disorders 2007; 9: 759-65.

Sun, Q, Ma J, Campos H, Hankinson SE, Hu FB. Comparison between plasma and erythrocyte fatty acid content as biomarkers of fatty acid intake in US women. Am J Clin Nutr 2007; 86: 74-81. 
Swierk M, Williams P, Wilcox J, Russell K, Meyer B. Validation of an Australian Electronic Food Frequency Questionnaire to Measure Polyunsaturated Fatty Acid Intake. Nutrition 2011; 27: 641-6.

Van De Rest O, Geleijnse JM, Kok FJ, et al. Effect of fish oil on cognitive performance in older subjects: A randomized, controlled trial. Neurology 2008; 71: 430-8.

Vandal M, Freemantle E, Tremblay-Mercier J, et al. Plasma omega-3 fatty acid response to a fish oil supplement in the healthy elderly. Lipids 2008; 43: 1085-9.

Whalley LJ, Deary IJ, Starr JM, et al. n-3 Fatty acid erythrocyte membrane content, APOE $\varepsilon 4$, and cognitive variation: An observational follow-up study in late adulthood. Am J Clin Nutr 2008; 87: 449-54.

Whitehouse P, George D, D'Alton S. Describing the Dying Days of "Alzheimer's Disease". J Alzheimer's Dis 2011; 24: 11-3.
Yanagisawa N, Shimada K, Miyazaki T, et al. Polyunsaturated fatty acid levels of serum and red blood cells in apparently healthy Japanese subjects living in an Urban Area. Journal of Atherosclerosis and Thrombosis 2010; 17: 285-94.

Yurko-Mauro K, McCarthy D, Rom D, et al. Beneficial effects of docosahexaenoic acid on cognition in age-related cognitive decline. Alzheimer's and Dementia 2010; 6: 456-64. 